This paper is published on January 09, 2023, as Chen et al., Sex inequality driven by dispersal, Current Biology (2022), https://doi.org/10.1016/j.cub.2022.12.027, and is available Open Access here: https://www.cell.com/currentbiology/fulltext/S0960-9822(22)01963-7

\title{
Sex inequality driven by dispersal
}

Yuan Chen ${ }^{1}$, Erhao $\mathrm{Ge}^{1}$, Liqiong Zhou ${ }^{2}$, Juan $\mathrm{Du}^{2}$, Ruth Mace ${ }^{1,3,4^{*}}$

1Department of Anthropology, University College London, 14 Taviton Street, London WC1H OBW, U.K

${ }^{2}$ State Key Laboratory of Grassland and Agro-Ecosystems, College of Ecology, Lanzhou University, 222 Tianshui South Rd, Lanzhou, Gansu Province 730000, PRC (People's Republic China)

${ }^{3}$ Institute for Advanced Study in Toulouse, Université de Toulouse 1 Capitole, 31080 Toulouse Cedex 06, France

${ }^{4}$ Lead contact

${ }^{*}$ Corresponding author: Ruth Mace.

Email: r.mace@ucl.ac.uk

\section{Summary}

Inequality between the sexes is pervasive both outside and inside the home. One contributing factor could be the dispersal of one sex at marriage that sets up sexspecific differences in relatedness to the group. Here we exploit the ecological

diversity and different social structures found in southwest China to investigate the role of sex-biased dispersal on inequality in the sexual division of labour. We use 
a wearable fitness tracker and validated readings by confirming that participants' daily 'steps' were positively correlated with time spent in high-energy activities, such as agriculture and animal husbandry work, and negatively correlated with low-energy activities, such as leisure and relaxation. We applied multilevel comparative approaches to examine the relative workload pattern between the sexes under different dispersal states. Our results reveal two characteristics that lead to an unfavourable division of workload: being female and dispersing at marriage. This is consistent with the hypothesis that males have increased bargaining power when remaining in their natal home, leading to inequality in workload.

Keywords: Post-marital residence, accelerometer, sex inequality, workload, labour division. 


\section{Introduction}

Deriving from Hamilton's kin selection framework, individuals are more ready to help their kin than strangers, other things being equal ${ }^{1,2}$. Yet kin competition may also exist and limit the evolution of helping behaviour intra-household, especially given asymmetric relatedness between sexes ${ }^{3-11}$. Dispersal is one such population-level process, which is believed to influence the resolution of family conflict and generate kin-relatedness asymmetry ${ }^{6,12}$. The costs and benefits of dispersal may depend on the cooperation and competition between ego and coresiding kin ${ }^{8}$. Consequently, helping behaviour may be sex-specific ${ }^{13-19}$. Children may be viewed as shared goods whereby both parents receive equal genetic benefits, yet one parent often invests more heavily than the other ${ }^{20}$. An evolutionary ecological perspective predicts that males and females may have distinct priorities regarding mating and parental strategies, and bargaining power may determine how much each sex contributes to the household ${ }^{20,21}$. Here we measured the division of labour in a natural context, across a range of populations and cultures, to test the hypothesis that sex and dispersal status are related to the balance of workload between the sexes.

Hunter-gatherers show a clear sexual division of labour between hunting and gathering, often associated with mating strategies ${ }^{22,23}$, though recent archaeological evidence from the Late Pleistocene suggested that females may 
also have participated in early big-game hunting 9000 years ago ${ }^{24}$. In agricultural and pastoral societies, the division of tasks between sexes is more variable. Based on visual scans, hunter-gatherers in the Philippines showed roughly equal amounts of leisure time between men and women, but the balance became more detrimental to women as 'off-camp' work increased ${ }^{25}$. Amongst farmers, males and females share numerous activities, although women often contribute more time and energy to domestic chores, animal husbandry, agriculture, and other family activities, whilst men's contribution is more limited to agricultural activities 26-29.

Most work on sex differences in workload has used visual scans and time budget data ${ }^{28}$. Technological advances are now allowing more detailed data collection on physical activity and work burden. Using accelerometers, studies on human health tend to focus on modern urban contexts, where activity is beneficial for older people ${ }^{30-32}$ and children ${ }^{33-35}$ in environments where obesity is a risk. However, in high workload contexts, a heavy disparity in workload can be harmful to human health ${ }^{36}$, especially for women ${ }^{37,38}$. Women worldwide enjoy less leisure time, involving more unpaid work as previous studies stated, particularly when multitasking ${ }^{39}$, which can be responsible for fertility decline ${ }^{40}$. Accelerometer data predicts human motion and has been validated statistically ${ }^{34,41-44}$. However, most such work has been done in the lab ${ }^{35,41}$; very few studies are in a free-living 
environment ${ }^{45}$, and even then, most focus on specific tasks ${ }^{46,47}$. There is not much research in agricultural and animal husbandry societies using this technology that compares both sexes across all kinds of physical activity in ecological contexts where high physical activity levels are indispensable to survive and reproduce. Despite the centrality of physical work in everyday life and the strikingly gendered nature of work in most populations, theories of how family structure influences the sexual division of labour are not well developed.

Social structure and the sex-specific demography of populations may influence the residential group's relatedness, hence determining the costs and benefits of helping household members of different ages and sex ${ }^{3,7,48-50}$. Dispersal at marriage, which generates different post-marital residences, is therefore likely to have an impact on the cooperative division of labour in the household ${ }^{51-54}$. It is possible that dispersal away from kin reduces cooperation and hence work for the household, or alternatively, the lack of kin support may reduce bargaining power and thus increase workload.

Here we collected accelerometer and time budget data on workload from 561 people from a range of rural households in southwestern China. Various ethnic groups inhabit the same ecological area, but their kinship systems and residence patterns are diverse. We investigated ethnic groups located in Sichuan Province and Gansu Province. Figure 1 shows the locations of the Han, Yi, Mosuo, Zhaba 
(also known as Zaba), zhAmdo, and mqAmdo populations. In China, Mosuo and Zhaba farmers are the only remaining matrilineal societies that still practice 'Visiting Marriage' (also called 'duolocality' in anthropology) ${ }^{55}$, which refers to couples staying with their birth family and living apart from their partner after pairbonding, which is extremely rare globally. In matrilineal groups, houses and farmland are usually inherited down the female line. The other four ethnic groups are patrilineal, of which the Han and Yi are mostly agriculturalists, whilst zhAmdo and mqAmdo people are nomadic or agropastoralists. In patrilineal groups houses and farms are usually inherited down the male line. Marriage bonds are weaker in matrilineal groups ${ }^{56}$, because, for a woman living with her natal family on her own family farm, the consequences of divorce are less costly than for a woman who is living on her husband's family farm and is reliant on her husband's property. However, these systems do have diverse residence patterns both within and between groups. Patrilocality (females disperse to live with their husbands' families), matrilocality (males disperse to live with their wives' kin), and neolocality (both sexes disperse at marriage) are residence patterns all followed by some families in the patrilineal groups (see Table 1).

This diversity provides a 'natural laboratory' to examine who benefits most from different kinds of dispersal at marriage, with the additional advantage that the data 
is from multiple villages and locations where the same team collected the data within one country. We exploit the ecological diversity and different social structures found in southwestern China to examine whether sex-biased dispersal is a disadvantage to the dispersing sex.

Technological advances arising from wearable fitness tracking enable us to address these questions on a much larger scale than was previously possible. The accelerometer data provides accurate measures of work rate. The time budget data, whilst a more subjective measure based on recall, gives insights into how the emerging differences in workload arise. This is the first large-scale comparison across different ecologically relevant contexts that examines how workload (overall physical activity) is determined by sex-specific demography and dispersal.

\section{Results}

Our analysis revealed that two main factors are associated with an unfavourable division of workload: being female and dispersing away from one's natal home.

\section{a) Differences in overall workload by sex and age}

Our results show that females took more steps than males per day across these six ethnic groups (Table S4). There was no sex difference in age distribution or household size, indicating no sample bias (men: $n=267$ observations; mean age 
$=48.74 ;$ s.d. $=13.63 ;$ women $=294$ observations; mean age $=47.29 ;$ s.d. $=13.27$; also see Table S4). Women walked at an average step count of $12045.78 \pm$ 2748.53, while men walked $9026.26 \pm 2997.40$ - after we applied multilevel model approaches with the ethnic group as a random effect, and the estimates of all multilevel candidate models indicate robust sex difference in the overall daily labour effort, adjusting for body mass index, any removal of the activity tracker, and being under unusual conditions during pregnancy, lactation, and illness (Table S6).

Our response variable, step counts per day, the proxy of overall daily workload formed an age-dependent inverted U- pattern between sexes, as individuals grew older, their workload increased to reach a peak around their later mid-life, followed by a gradual decline until end of their lives, presented by raw person-level and daylevel data (Figure S2). We generated multiple days of step count data from the same person based on their full days wearing the accelerometer (days worn) and performed multilevel analysis for the day-level activity data $(n=640$ days). As seen in Figure 2, individuals aged over 60 made a labour contribution of 4578 fewer daily step counts than those aged 30 to 40 and 3386 fewer than those aged 40 to 50 . There was no substantial difference between the oldest group and age group of 50 and 60. Model comparison results are displayed in Supplementary information (Table S6). The age differences remain robust to days worn, 
suggesting consistency in day-level data and person-level data (Figure S3). More days worn correlated with a less daily work effort, implying a complementary labour pattern over consecutive day. Nevertheless, this effect is not significant.

Overall, the multivariate multilevel model provided robust evidence of substantial differences between sex and age groups among these ethnic groups and also with various forms of social organization. These differences persist strongly even after controlling for the biological factors of BMI or other unforeseen environmental factors, such as removing the activity tracker during participation in the study.

\section{b) Differences in workload by social organization}

Households with more than eight people had an average step count of $9547 \pm$ 2982, while individuals in the smaller households took $11450 \pm 3304(p<0.001$, Figure $3 \mathrm{~A}$, multivariate multilevel modeling). Household size is significantly negatively correlated with daily step counts among candidate model lists, but the contribution to daily workloads is very small compared to other covariates in the model (Figure S3 and Table S6).

Some groups have duolocal residences, and men under duolocality usually live separately from their spouse and their biological offspring. Duolocal households tend to be large, whereas neolocal couples tend to have the smallest household 
sizes (Table S4). Our data also show that duolocality is more prevalent among younger couples (Table S4) ${ }^{8}$.

By comparing the differences in step counts between the four post-marital residence patterns, we discovered that neolocal couples (both sexes disperse) take the most steps, while duolocal 'walking marriage' couples (neither sex disperses and spouses live apart) take the fewest (see Figure 2, and Figure 3B, predicted from the best-fitting model). This implies that men's activities are mostly responsible for the differences. Only in matrilocal households (males disperse at marriage, while females stay) were there no significant sex difference in the number of steps, which is the only post-marital residence pattern in which men disperse to live with their wife's kin (Figure 2).

As an alternative way to measure post-marital residence, we also explore individual dispersal status. We find that individual dispersal status and age group and sex remain important (Table S6). Body mass index (BMI), removing activity trackers, lactation or being unwell, and household size are all insignificantly negatively correlated with daily step counts (Table S6). We tested models with and without a sex-dispersal interaction: when both main effects of sex and dispersal are present, the interaction is not significant $(b=651.11,95 \% \mathrm{Cl}[-$ 1988.30, 3290.51]; also see Table S6), implying that dispersal is a disadvantage independent of sex that is driving individual workload variation. The dispersal 
model and the post-marital residence model had no significant differences $(L R=$ 2.02, $P=0.73$, ANOVA). Furthermore, separating the married sample by sexes revealed significant differences between dispersing and non-dispersing men $(b=$ 1865, SE $=941,95 \% \mathrm{Cl}[47,3684])$, but not between women $(b=1199,95 \% \mathrm{Cl}[-$ $763,3.16])$. This suggests a greater disadvantage of dispersal for men. Figure 4 shows a direct view of the interaction between personal dispersal status and sex, where the model revealed that being female and dispersing are the two key characteristics contributing to greater workload (daily step counts) (Figure 4).

The increase in the workload of dispersing individuals, compared to those living in their natal households was about 2000 steps per day $(b=2069.67,95 \% \mathrm{Cl}$ [798.54, 3533.84], multilevel modeling). Controlling for days worn, dispersing individuals also took significantly more steps than non-dispersing individuals $(b=$ 1947.11, 95\% Cl [647.16, 3247.06], multivariate multilevel modeling). The bestfitting model predicted that dispersing group walked $11749 \pm 3165$ steps per day ( $n=354$ days of observations), while non - dispersing group walked at an average of $9935 \pm 3040$ steps daily ( $n=286$ days of observations); Additionally, dispersing males worked more compared to non-dispersing males (Dispersing men: $n=113$; Mean $=10030 ;$ Not dispersing men: $n=165$, Mean $=8370.34 ; b=1801.94,90 \%$ $\mathrm{CI}[232.20,3371.67]$, multilevel modeling). Overall, these results reveal the role of 
dispersal in driving sex inequality and possibly explain why globally males rarely disperse.

\section{c) Differences in activity budgets by sex}

In addition to the data from the accelerometer, we collected time budgets by recall over the last 24 hrs. Whilst this is a more subjective form of data, it enables us to see how it is that some of the differences in activity that we have described may emerge.

Females are more active and spend more time than males in all work intensities of agricultural and animal husbandry, according to self-reported time budgets (Figure 5). Females spent significantly more time than males doing moderateintensity domestic chores like cooking, laundry, and sweeping (Mean(male) $=$ 18.247 , Mean $($ female $)=88.402$ ), as well as some moderate-intensity agricultural and animal-husbandry work like cow feeding and milking (Mean $($ male $)=59.584$, Mean $($ female $)=91.243)$. Women also appear to spend more time on 'Gathering' activities $($ Figure 5, Mean $($ male $)=11.673$, Mean $($ female $)=20.612)$; This is largely mushroom collecting on the peaks of high mountains, which is a traditional, energy-intensive manner of supporting a family that mainly occurs in Zhaba areas 57. Men (Mean age $=45.87, \mathrm{~N}=267$ ) in our sample are more engaged in building and entertainment than women (Mean age $=44.96, N=294$ ), and they reported spending a lot of time playing with their cellphones and watching television. Men 
with duolocal post-marital residence are involved in lots of political activity and social engagement ${ }^{49}$. When controlling for any broad cultural difference among these populations, the sex difference in time spent working remains robust (Figure S4).

By setting up multilevel models, our data confirmed the previous linear regression results that women are significantly more dedicated to both intra-household work and out-of-household work (Figure 5 and Figure S4). Three separate categories of work referring to wage labour indicate no sex difference (Figure S4, activity codes for wage labour work: AT8 - AT10). Combining the result of substantial sex differences in household production and marginal effects of sex on wage labour supported our detailed twenty-four activity-category design. Significant sex differences are seen in house construction, even though the interpretation of this should be careful, as residents also construct houses for other families, as a traditional form of labour exchange. Therefore, house building may serve as a status or social signal. We also show that men spent more minutes on self-care (AT17), amusement (AT20), driving (AT21 and AT22), and other activities (AT24) (Figure S4) than women. 


\section{d) Sex difference in energy consumption among light-intensity, moderate- intensity, and high-intensity work.}

Using the intensities of physical activities, we established a crude classification for these 23 categories (the 24th is other activities, see Activity Code in Table S7) into three groups: light-intensity physical activity (LMET), moderate-intensity (MMET), or high-intensity physical activity (HMET), after Ainsworth, 2000. The metabolic equivalent task levels (METs) are a general indicator of metabolic consumption and physical activity energy use ${ }^{58}$, referring to the ratio of the energy expenditure rate for activity compared to resting energy expenditure. The comparative outcomes of ego's percentage of time spent in light-intensity (LMET), moderateintensity (MMET), and high-intensity (HMET) activities show that men devote more time to LMET activities (Figure S5), whilst women devote more time to HMET activities (Figure S5); there was only a minor sex difference in time spent in moderate activities (Figure S5).

The relative difference in time allocation of diverse activities defined by their physical activity level among ethnic groups and sex agreed with results from the accelerometers. Ethnic groups were included as covariates, and villages were treated as random effects, which is consistent with previous model construction in predicting daily steps taken. Men's time spent in activities of high intensity is highest in the Han group (who are mainly neolocal), while women's highest time 
contribution of $\mathrm{HI}$ work is found in Zhaba (who are mainly duolocal) (Figure S5). Amdo women (who mainly live in patrilocality) are less likely to be involved in work of light intensity than are men. Further, our data suggested that the dispersers appear to be more engaged in vigorous-intensity activities than those who stay with their natal family, although the effect does not reach statistical significance.

Men aged 30 to 40 appear to spend higher percentages of their time on vigorousintensity work than other age groups, and they do less light-intensity activity (Figure S5). Women aged 40 to 50 have a greater participation rate in highintensity work compared to the oldest group aging over 60 (Figure S5). Our data show women's workload is more age-dependent, while men's time allocation is more dependent on the intensity scale of physical activities.

This further analysis of the pattern of energy consumption revealed consistent sex differences in time budgets across the different populations that support the conclusions of sex differences in overall activity found in the accelerometer study. 


\section{Discussion}

Our study demonstrates that sex differences in work burden are substantial in these farming and herding communities. Workloads in this region are high. Women, in particular, got up extremely early in the populations we studied, notably for milking; they had to feed livestock and prepare food, and gather cow or yak dung for heating and cooking. Independent of the post-marital residence (dispersal) pattern, females do more household and more subsistence activities (farming, husbandry work, gathering work) than males, whereas males are engaged in more building work and leisure. This generally results in a workload penalty for women. Men may have more bargaining power simply by being the physically stronger sex. Sex differences in parental investment are strongly influenced by the mating system ${ }^{21}$. While not all the work done here is necessarily a parental investment, farming and domestic chores are a large part of the workload, and both these tasks contribute directly to children's growth and wellbeing ${ }^{59}$. Sexual conflict means that men can often benefit from investing in themselves, referring to private gains ${ }^{23}$, or seek mating opportunities elsewhere, leading to less family investment ${ }^{49}$; this may be contributing to them doing less work overall. 
The magnitude of this gendered imbalance is further determined by the social structure of the household. We find that those who disperse at marriage, be they male or female, suffer an additional penalty, doing more work than those who stay in their natal home. Men may choose not to disperse so as to reap the benefits of proximity to their kin and their social networks in their natal areas. There is an equal gender balance in work only if married males have dispersed to live with their wives' natal families. Relatedness to individuals in the household may influence negotiating power ${ }^{70}$. The cost of dispersal could be high at first; however, dispersers benefit from increasing relatedness to the household as they age because they produce more offspring in their new household $9,60,61$. Male dispersal may be rare globally because married men do more work under matrilocal (i.e. uxorilocal) post-marital residence; this fits with accounts from Malawi of men living in matrilineal households being expected to work hard on their wives' farms ${ }^{62,63}$.

Men do least under the patrilocal and duolocal post-marital residence, where the men are living in their natal home. Men appear to be drawing on the benefits of high relatedness to their natal household to reduce their workloads in these households as predicted. However, females in duolocal households, who are surrounded by their matriline and highly related to all the other household members, work hard relative to their brothers. Duolocal residence is hard to compare directly with the other groups as this is the only case where husband and 
wife live apart (in 'walking marriage'), sisters are reproducing communally, and men live with their sisters' children rather than their own children ${ }^{64}$. Because the children of the men reside elsewhere (due to 'walking marriage'), this leads to asymmetries in male and female relatedness to the household ${ }^{7}$ generating sexspecific differences in competition between siblings. Mothers may invest more in sons compared to daughters due to reproductive competition between their coresident daughters, but not their sons whose children are in other households, as we have discussed elsewhere ${ }^{49,65,66}$. Theory and data suggest other duolocal social systems in the animal kingdom, such as resident killer whales, are also characterized by mothers preferentially helping their male rather than their female offspring ${ }^{67,68}$.

Middle-aged people's high workloads may reflect their increased relatedness to the household as they get older ${ }^{7}$, in line with predictions on kinship dynamics ${ }^{9}$. The other explanation is middle-aged people may be indirectly investing in grandchildren, alleviating the workload of their reproductive daughters or daughters-in-law who are breastfeeding and caring for young children.

Additional explanations for sex-specific dispersal include that it may have been driven by warfare and intra-family conflict ${ }^{17}$. Micheletti et al found that the sex that competes more locally is more likely to be altruistic, whereas the sex that competes more globally is favoured to receive altruism from both sexes ${ }^{15}$. In the case of 
patrilocality, 'boys' clubs', where both males and females preferentially help males, can be generated ${ }^{15}$. Females compete for reproductive resources locally in matrilineal groups, potentially explaining why we observed a high prevalence of cooperative females under duolocal residency.

The sex difference in our farming and herding sample contrasts with results in hunter-gatherer populations that have shown that males walk over 18000 steps per day on average, more than females who take 10921 steps on average ${ }^{69}$; and males spent more time in forest work among agro-pastoralist in Nepal ${ }^{28}$. Our results also differ from findings among Hadza foragers in Tanzania, where fat-free mass was the strongest predictor of TEE (Total energy expenditure), but in studies that took fat-free mass into account, there was no relationship between TEE and sex, physical activity, or workload ${ }^{70}$. In a recent study on physical functioning in Hadza foragers nor Pokot pastoralists in East Africa, sex was not correlated with time spent in MVPA (moderate-vigorous physical activity) ${ }^{71}$. In an industrialised setting, the US, males took more steps than women (5340 vs 4912 steps per day) 72. A cross-sectional study focusing on 46 countries, studied the association between obesity and activity inequity using smartphone data, and they found that the Chinese walked over 6000 steps on average daily, while South Africa, Indonesia, and the Philippine people walked less than 4500 steps, and American adults walked 4774 steps on average ${ }^{73}$. They discovered obesity was negatively 
correlated with activity per day in this sample of mainly high-income countries (where physical workloads are lower). Evaluating the relationships between workload and health is challenging, but in this context of high work burdens, it can be hypothesized that time spent on leisure is associated with increased fitness, but time spent on arduous subsistence work is associated with increased health risk. The interpretation of self-reported time-budget data should be cautious. However, the differences between our cross-cultural data from farmers and herders and hunter-gatherer data provides insight into the likely association between subsistence systems and sex differences in workload patterns in human communities.

Group living represents a puzzle when there is reproductive competition between co-residents ${ }^{74}$. Communal living and breeding are often interpreted as resulting from a constraint on breeding opportunities or living space ${ }^{75}$, but our results suggest it may also generate efficiencies. The reduced workloads in large farming households suggest economies of scale, and this increased efficiency may underpin why group households form in societies such as the duolocal Mosuo and Zhaba.

In conclusion, we discovered that being female and dispersing at marriage are two factors that contribute independently to an unfavourable division of labour. This suggests dispersal away from kin appears not to reduce co-operation; rather the 
lack of kin support reduces bargaining power. Males in particular appear to have greater bargaining power when they stay in their natal family. Leaving the immediate help of family appears to raise the relative work burden in domestic life. This may explain why males rarely disperse. Female dispersal at marriage is the most typical pattern of dispersal globally ${ }^{52}$, leaving most women shouldering the double burden of their sex and their post-marital dispersal contributing to gender inequality in workload.

\section{Strengths and limitations}

The research design minimizes response and sampling bias in a cross-cultural quasi-natural setting, collected across many villages and locations with diverse social structures within one country. Data are obtained independently from reported time budgets and activity trackers, presenting consistent results. The comparative nature of the data warrants the conclusion that being female and being the dispersing sex both mean you work more. Compared to observational scans, the step counts, equivalent to 1440 minutes per day, validated by time budget, enable us to fully capture the full activity expenditure of individuals, not only during the day but also at night.

One important limitation of this study is the association between workload and fitness is hard to resolve. Step counts per minute associated with walking at 3 metabolic equivalent tasks (METs) differ between sexes, which may contribute to 
part of the sex difference in our study. Other studies have found men took more steps than women to reach an equivalent energy consumption per minute ${ }^{46}$.

Time-budget data also revealed that men spent more time in activities of light intensity, whereas women took more time on activities of moderate and vigorous intensities from a set of multivariate multi-level models. The percentages of time spent on each activity of various intensities appear to be sex-specific and depend on ethnic group. Combining these activities and simplifying categories based on the intensities of time-budget data by splitting them into three categories must be treated with caution, as it is a crude measure. ${ }^{14}$ Our workload proxy is a component of both offspring care and a contribution to personal subsistence, constraining our interpretation of the scale of offspring provisioning.

Sex-biased dispersal has been discovered to influence parental care in animals; specifically, the more philopatric sex are found to provide more care under sexual conflict ${ }^{14}$. Here, we present evidence of the contribution of dispersers intrahousehold is higher, probably because more time is spent by dispersers on more vigorous-intensity work. Further study could use more detailed and focused variables to disclose the bargaining power between sexes within-pair. 
Acknowledgments. We thank the local people for their participation in this research and their great help and assistance in fieldwork. We thank Jiajia Wu, Yuping Yang, Bai Peng-peng, Almira Anwar, Yu Tang, Xin Zhu, Gang Jin, Hanzhi Zhang, Yishan Xie for help with data collection. We thank Bin Liu, Guo-Song Gao, Shi-Liang Li, Feng-Yi Xu, and other programmers from Huami Company for their immense help in programming and technical support. We are also grateful to two anonymous reviewers, Andy Gardner, Alberto Micheletti, and Mark Dyble for their great comments. We also thank the Human Evolutionary Ecology Group at UCL. This work was funded by the European Research Council (Grant no. 834597, Evobias: 'Sex-specific demography and the evolution of gender-biased harmful cultural practices'), University College London, and by Lanzhou University.

Author Contributions: $\mathrm{R} \mathrm{M}$ conceived the study. Y C carried out the analysis and modeling work. Study design and implementation by $Y C, R$ M. Data collection by Y C, E-H G, L-Q Z, J D. Writing by Y C and R M. All authors gave final approval of the manuscript.

Declarations of Interest: The authors declare no competing interests. 


\section{Main-text figure/table legends}

Figure 1 Location map of the study areas in southwest China. ZhAmdo, mqAmdo, Zhaba, Mosuo, Han, and Yi represent six ethnic groups. On the map of China, the four field sites-Zhuoni County (zhAmdo), Maqu County (mqAmdo), Daofu County (Zhaba), and Yanyuan County-are highlighted in green (mixed population co-reside here). Interviews with Mosuo, Han, and Yi took place in the same ecological setting in Yanyuan County's Lugu Lake area. Southwest China is depicted on the map of China in the inset plot as a squared black box. See also Table S3.

Figure 2 Forest plot of generalized linear mixed model estimates shows how many step counts vary with post-marital residence pattern (PMR) for 566 married adults' daily observations. Control variables included whether they removed the wristband at any time, breastfeeding or other unusual situation, body mass index (BMI), and age cohort. Duo: Duolocal residency, neither sex disperses after marriage. Mat: Matrilocal residency, the husband disperses to live with his wife's kin. Neo: Neolocal residency, both sexes disperse after marriage. Pat: Patrilocal residency, the wife disperses to live with the husband's kin. Village ID was set as a random effect. Significance: $p<0.001^{* * *}, p<0.01^{* *}, p<0.05^{*}$. See also Table S4, S6, and Figure S3.

Figure 3 Adjusted workload pattern predicted from GLMM varies by post-marital residence pattern and household size across a different social organization ( $\mathrm{N}=566$ days of observation); (A): Steps and household size pattern. (B): The effect of sex and place of residence on workload (steps). Small indicates a household with less than 4 residents; Medium indicates 4 8 residents, while four and eight residents are included; Big indicates more than eight residents in the household. 'Duo' indicates duolocal residency (both sexes stay at their natal birthplace and practice the 'walking marriage'), and 'Mat' denotes matrilocality (males disperse to live with their wife's kin); 'Neo' refers to neolocal residency (both sexes disperse at marriage); 'Pat' means patrilocality (females disperse and live with their husband's family after marriage). See also Table S4 - S7. 
Figure 4 Interaction effect between sex and individual dispersal status ( $N=640$ days). See Table S6.

Figure 5 Time spent on work of different intensity by sex. Mean minutes spent in (A) household chores and (B) subsistence work by sex $(\mathrm{N}=561)$. LI: Low-intensity work; MI: Moderate-intensity work. HI: High-intensity work. AAAH work represents Agriculture and animal husbandry work; Gathering: adopting mushrooms at the top of high mountains. Blue indicates males, and red indicates females. Standard errors are displayed. See also Figure S4 - S5 and Table S7.

\begin{tabular}{lllll}
\hline Ethnic group & Matrilocal & Patrilocal & Duolocal & Neolocal \\
\hline ZhAmdo & 13 & 35 & 0 & 0 \\
Han & 1 & 28 & 0 & 22 \\
Mosuo & 15 & 14 & 51 & 24 \\
MqAmdo & 13 & 34 & 0 & 9 \\
Yi & 6 & 66 & 0 & 14 \\
Zhaba & 28 & 16 & 77 & 3 \\
\hline
\end{tabular}

Table 1 Sample distribution of those providing time-budget data by post-marital residence for the six ethnic groups. We categorized post-marital residence for married people into matrilocal (male disperse), patrilocal (female disperse), duolocal (neither sex disperse, a couple lives apart), and neolocal (both sexes disperse) after marriage ( $n=469$ people, people-level data). Among these people, 438 currently married people were available for workload analysis, providing 640 full days of accelerometer data. See also Table S4 for day-level sample distribution and Figure S2 for detailed descriptive plots. 


\section{STAR * METHODS}

Detailed methods are provided in the online version of this paper and include the following:

- RESOURCE AVAILABILITY

- Lead Contact

- Materials availability

- Data and code availability

- EXPERIMENTAL MODEL AND SUBJECT DETAILS

○ Study sites

- Validation of workload data

- The relationship between step counts and workload

- Comparative data collection

- METHOD details

- Validation of activity tracker in the lab.

- Validation of activity tracker in the field.

- Model validation of step counts

- QUANTIFICATION AND STATISTICAL ANALYSIS

- Generalized multilevel comparative analysis

\section{RESOURCE AVAILABILITY}

Lead contact

- Further information and requests for resources and reagents should be directed to and will be fulfilled by the Lead Contact, Ruth Mace (r.mace@ucl.ac.uk).

Materials availability

- This study did not generate new unique reagents.

Data and code availability 
- The de-identified demographic data have been deposited at UCL Research Data Repository, and accession numbers are listed in the key resources table. They are available upon request if access is granted, to request access, contact UCL. In addition, the summary statistics describing these data derived from these data have been deposited at UCL Research Data Repository.

- All original code has been deposited at Github https://github.com/YUANDALAO/activity-tracker.git and is publicly available as of the date of publication.

- Any additional information required to reanalyze the data reported in this paper is available from the lead contact upon request.

\section{EXPERIMENTAL MODEL AND SUBJECT DETAILS}

\section{Study sites}

We conducted a demographic survey over six rural populations in western China, Sichuan Province, and Gansu Province (see Supplementary Information and Figure 1). All individuals gave their consent, and the People's Govt at each local site permitted this research. This research was approved by Lanzhou University (Dept of life sciences) and the UCL (University College London) Research Ethics committee (no 0449/003).

Study sites were selected to encompass a range of social systems, including groups with both high and low levels of female dispersal at marriage ${ }^{6,76}$. Patrilocal groups have high levels of female dispersal and are mostly patrilineal (wealth inheritance goes down the male line); whereas duolocal groups have low female and low male dispersal and are matrilineal (wealth inheritance down the female line), and spouses often live apart (Table S3 give basic population details). However, within each ethnic group, there is also diversity in residence patterns, including in most groups some neolocal households where both sexes disperse. For each household, one adult representative was interviewed about the personal information of all family members, which included name, ethnic group, gender, year of birth, marital status, and dispersal status (see details of Maqu, Zhouni, Lugu Lake and 
Zhaba study sites and samples in the Supplementary information Table S3). The numbers following each of the residence patterns after marriage both within and between ethnic groups are shown in Table 1.

\section{Overview of the activity tracker (Miband 2)}

Studies of wearable activity trackers have not found robust evidence to indicate one model is better than others in terms of accuracy among Lexin, Miband, Fitbit Charge, and other pedometers ${ }^{77}$. We compared Miband2 and Fitbit as alternatives, selected for both practicality and technical support ${ }^{77}$. Some studies suggest that Miband2 is not intensely stable, compared with Fitbit, as Miband2 mainly relies on arm swing ${ }^{47}$. However, activities such as milking which includes moments without walking to produce energy expenditure are also included in our research. Compared to Fitbit, we observed that Miband2 is more stable with the lowest variation coefficient when multiple trackers were subjected to the same activity in a test (see Table S1).

Step counts are raw activity data derived from arm movement, whereas some studies use activity counts (AC) calculated depending on combining the basal metabolic rate and step counts. Previous studies revealed that activity counts might be inappropriate for studying physical activity since they underestimated the daily physical activity energy expenditure (PAEE) when activity counts were converted into kilocalories per kilogram per day ${ }^{77}$. Furthermore, a recent study presented that total energy expenditure (TEE) is independent of body fat, and they found no significant within-individual, among-individual correlation between TEE and body fat ${ }^{78}$. The purpose of this study is to investigate the total raw activity amount individual performed during a day, rather than how many calories they burnt throughout each task. Thus, we use Miband2, and its daily 'step' counts as the proxy of workload for this comparative study in rural areas under different socioecological contexts.

\section{The relationship between step counts and workload}

In the laboratory-based experiment, we investigated the relative validity of Miband 2 and Fitbit 2 among four subjects (Table S1). Activity counts were substantially associated with PAEE (physical activity energy expenditure) 
and EE (energy expenditure), as in prior studies ${ }^{77,79,80}$. Activity counts (AC) values measured from most accelerometers and PAEE have correlation coefficients of over 0.9077 . However, rather than energy expenditure (calories consumed during work), the goal of this study is to investigate the workload pattern between populations. Although sedentary behaviour has caused public health concerns in metropolitan regions, in our rural field sites, local inhabitants are active or vigorously active for the sake of survival under harsh ecological constraints. Since time allocation during lifespan is a constant consideration from evolutionary perspectives, especially when deciding between mating and household maintenance. However, the time budget raised many concerns about the underestimation of higher PAL. In particular, a slow-moving person may spend more minutes on a single task and hence expend less energy overall than someone with higher METs, bringing the benefits of accelerometers into public awareness.

Accelerometers function by integrating an accelerometer signal over a predetermined time interval commonly referred to as an epoch ${ }^{77}$. In the case of our activity tracker, our epoch is a one-minute interval, making it difficult to use the epoch cut points to calculate the amount of time spent at various degrees of intensity, as the choice of epoch length will have a substantial impact on the study results. Because this is a cross-region study involving people from distinct subsistence systems, inhabitants wake up at different hours and begin their workday at different times, consequently, they stop at various times for each activity. As a result, deciding the time cut point is practically difficult. Since the purpose of this study was to examine activity patterns over a few days across populations, the precise length of the epoch is not critically important and hence, the default setting, a 1-min epoch, was used. Furthermore, we sampled these populations during the same busy season and recruited the participants to wear the activity tracker for 2-5 days to avoid environmental restrictions. As a result, the overall quantity of workload per day in our study is calculated by adding up the 1-minute interval step count to 24 hours or 1440 minutes in total.

\section{Comparative data collection}

We conducted a questionnaire data on-time budgets and activity across a total of 55 villages at six different sites in western China in 2017 and 2018 
(see supplementary material Table S3 for details of the sample). 561 people from different ethnic groups completed the time-budget questionnaire (62 questionnaires were collected from the Zhuoni area, 64 from the Maqu area, 275 around Lugu Lake, and 160 from the Zhaba area), of which 294 were from women and 267 from men (see Table 1). The sample included individuals with four kinds of post-marital residence (see Table 1 for details): patrilocal (female disperse), matrilocal (male disperse) and neolocal (both sexes disperse), and duolocal (neither sex disperses with 'walking marriage', which is only found in Mosuo and Zhaba populations ${ }^{6-8,49}$ ). Across all these samples, we got data from 536 married people in total (see Table 1).

We obtained 561 people's activity data and time-budget data, after omitting invalid step count data, 536 activity data in the last $24 \mathrm{hrs}$ were left. Among all 561 individuals, 469 were married people with demographic data, and 438 of them provided activity data in the last $24 \mathrm{hrs}$ (Men $=194$, women $=$ 244). Six hundred and forty activity observations were provided by 438 married individuals. And we obtained 569 daily observations of 469 married individuals, down to 566 of 394 married people when constructed models with missing data omitted.

Some of those interviewed for demographic information were randomly asked to participate in the activity research. They were asked to wear a plastic removable accelerometer on their left hand (which we refer to as a wristband). Other adult family members in the same household at that time who also agreed to wear wristbands were also included in the study. Participants were asked to wear the wristband all day and night until we collected them 2-5 days later. That time span was chosen because a shorter time estimates the daily activity pattern of participants less accurately (for instance, on rainy days, people sometimes work much less); and after 5 days, the recovery rate declines, and the wristbands lose power or can be lost $32,46,81$.

On collection, we conducted a questionnaire on the last 24-hour time budget by the recall. This enabled us to validate the electronically collected data on activity by comparing it with the participants' account of their activity over the last day (see also Table S2). We asked if they had removed the wristband 
at any time. We measured the height and weight of participants in the activity study.

\section{METHOD DETAILS}

\section{Validation of activity tracker in the lab.}

Four participants performed a walking task on the same day, and everyone was asked to wear the two kinds of wristbands at the same time for twenty minutes. We selected 8 Miband 2 and 13 Fitbit Charge2 randomly and separated them into two sets (Set 1: Six Fitbit Charge2 + four Miband2; Set 2: Another seven Fitbit Charge2 and another four Miband2). And subject $A$ and subject $B$ were told to wear the same four Miband2 and seven FitbitCharge2 (Set 1) but in a different period, subject $C$ and subject $D$ wore the same four Miband2 and six Fitbit Charge2 (Set 2) but not the same period. And then we calculated the mean steps over the last twenty mins, standard deviation, variance, and coefficient of variance for each round by both bracelets (referred to as Miband2 and Fitbit Charge2) to measure the stability of each kind of activity monitor (Table S1).

\section{Validation of activity tracker in the field.}

For data validation of wristbands in the field, we randomly recruited three persons in one family to track their daily lives for 24 hours. When we began to follow them, the old male had not woken up, however, the two females (mother-in-law and daughter-in-law) had already started their work. (also see Figure S1).

The younger female's sleep time we recorded was at 10:10 pm and got up at 5:31 am, which was consistent with what the wristband sleep data showed. In general, females woke up earlier than males. Notably, this younger woman felt a bit unwell and rested at some point in the day. Hence, the workload on that day was lower than usual.

\section{Model validation of step counts}


Most previous studies of workload obtained activity information through selfreport 26,27,59,82. Although data obtained from questionnaires is effective, participants frequently ignore some light activity when self-reporting, and participants' physical, cognitive, and emotional conditions can all influence reporting. We used 24-hour recall to estimate time budgets to record time spent on each activity (see Table S7, Supplementary Information). Combined with the time-budget questionnaire, we used wearable activity trackers to provide more detailed information, including activity frequency, activity duration time, and 'steps' or amount of activity.

Studies of metabolic energy consumption have found that the costs of daily activities vary greatly. We recorded 24 categories of activity in total (see supplementary information: Activity code - Table S7) depending on different metabolic equivalent tasks levels (METs), which refers to the ratio of the energy expenditure rate for activity compared to resting energy expenditure, and we classified some subsistence-related activities into L.I. (Low Intensity, energy expenditure below 3 METs), M.I. (Moderate Intensity, energy expenditure between 3 METs and 6 METs), and H.I. (High intensity, energy expenditure above 6 METs) in our analysis abstracted from the Compendium of Physical Activities ${ }^{58}$.

Our activity tracker (Miband2, ${ }^{83}$ ) is programmed to record and save activity counts in 1-min intervals. In three ways, we validated our wristbands with workload data. To begin, although these activity trackers had been empirically tested before being released to the public, we placed all the activity trackers in the same sack and recorded every single step count after a given same journey, to examine carefully if they performed consistently, and then we eliminated the two outlier wristbands that were significantly different from the mean step counts. Secondly, we double-checked that the wristbands were recording data corresponding to our time-budget activity data. An example of the raw data from three individuals is also shown in the supplementary material (Figure S1). Lastly, we regressed total step counts as the dependent variable and the previous days' time allocation data as the predictive variable, using a multivariate permutation regression (Permutation tests, also called randomization or re-randomization tests) ${ }^{84}$. A few activities, such as teaching and studying (AT8, AT10, AT18, AT19), had a 
low participation rate; hence six activities were not included in this permutation test (see Table S2). Because step counts are related to height and weight, we made sure to account for that in subsequent analysis. Covariates, like weight and height, did have an impact on the step counts, whereas the results of the generalized linear regression model also revealed that the time reported by participants undertaking subsistence work and gathering (AT3, AT4, AT5, AT7, codes presented in Table S2) was significantly positively correlated with the total counts of steps. When individuals spent more time on self-care and leisure, they took fewer steps. Overall, these results are consistent with what we expect if the step count is an indicator of the individual's workload.

\section{QUANTIFICATION AND STATISTICAL ANALYSIS}

\section{Generalized multilevel comparative analysis}

Data analyses were completed by $\mathrm{R}$ software, in which the "ImPerm" package was used to build the prediction model and permutation test ${ }^{85}$, a non-linear logged multi-level mixed model was built in "nlme" ${ }^{86}$, the model screening process was completed in "MuMln" 87, and "ggplot2" 88, "sf" 89, "stargazer" 90 packages were used for figures and tables. In regard to timebudget data and activity data, all the categories of activities and step counts show an overdispersion when we conducted generalized multilevel Poisson models. Additionally, a series of Poisson models did not perform well, even after we applied Poisson distribution and log link for daily step count data, as well as binomial distribution and logit link for proportional time-budget data. Therefore, all the generalized mixed-effects models were achieved with the 'Ime' function in the 'nlme' package.

In the presence of a random effect, the generalized mixed multi-level model is an effective way to deal with the nonnormal data with a small sample size 86,91. Besides, by relaxing LMM's assumption that the dependent variable (and residuals) follows a normal (Gaussian) distribution and extending GLM's scope of inference beyond a single random population, GLMMs combine and extend the properties of LMM and generalised linear model (GLM) techniques ${ }^{92}$. 
Step counts display a slightly left-biased normal distribution (See Figure S2) despite the fact that it was analyzed as count data in most cases. Ives (2015) recently argued that transformed count data analyzed using LMMs can often outperform Poisson GLMMs ${ }^{93}$. Thus, we applied a transformation to step count data. We used a square root transformation for the dependent variable in all GLMMs (generalised linear mixed models) ${ }^{94}$, and we found a consistent outcome between the model of raw step count and of square root transformation (see Supplementary Information), thus model estimates of raw step counts as the dependent variable were shown in the Result section for direct model interpretation, and the transformed ones were displayed in the supplementary information. To explore whether there is a difference in labour (step counts) between individual men and women under the different post-marital residence patterns, we developed generalized linear mixed models for married people across the six ethnic groups ( $N=566$ observations); the village was set as a random effect to control for any broader ecological differences between field locations. All the models controlled for age cohort, whether individuals are under some unusual circumstances, e.g., lactating or ill $(\mathrm{Y} / \mathrm{N})$, whether participants ever took off the wristband $(\mathrm{Y} / \mathrm{N})$, and body mass index (BMI) ${ }^{45}$. In addition, the interaction between sex and post-marital residence (PMR) was added to the full model. Due to the non-linear correlation between household size and steps, a power function was needed for the household size variable after model comparison (Table S5). To discover how the relative sex differences in workload vary with the four types of PMR, sex was included as an interaction function with PMR. We did not find a significant association between days worn and step counts, thereby days worn were not included across other candidate models (see Figure S3).

To establish whether these sex differences hold when controlling for individual-level fixed effects (of age, sex, and whether an individual had taken off the activity tracker in the past day), we used generalized multilevel linear modelling to predict individual step counts throughout populations. The distribution of person-level activity data displayed similar results to daylevel observations, albeit a detectable difference was discovered between person-level and day-level data in the association between daily step counts and age groups (see Figure S2). 
The relative labour demand per household - and consequently the work burden faced by people from various households - can be determined by household size. Given the child policy restrictions, fertility varies only little between households, and a wide range of household sizes will largely reflect the number of adult co-residents. Instead of using the dependent-ratio variable, we used the household size in the following analyses. This is because the elderly in our study population performs a wide range of both intra- and extra-household chores, calculating a relative labor force demand by simply compiling the ratio of non-workers to labourers is not very meaningful. The data displayed no significant association between household size and sex (Est $=-0.1855,95 \% \mathrm{Cl}[-0.557,0.186]$, univariate multilevel modelling, day-level). Additionally, no significant linear association between household size and age range was discerned (Est $=-0.010,95 \% \mathrm{Cl}$ $[-0.024,0.005]$, univariate multilevel modelling, day-level). Our data, however, revealed that more individuals aged over 60 years were sampled among smaller households with (Figure S2, day-level), compared to other age groups. This suggests that there may be multicollinearity between household size and age groups when constructing models. Therefore, we compare model sets with and without household size, respectively (Table S6). Comparing models with different functional specifications for household size revealed that a quadratic model fits better than a linear model $(\triangle \mathrm{AIC}=$ $1, w=62.2 \%$ of model sets, $w$ : weights), but a quadratic model does not fit better than the cubic model in the multivariate models (Table S5). Therefore, the cubic function of household size is preserved in the best-fitting model.

\section{References}

1. Axelrod, R., and Hamilton, W.D. (1981). The evolution of cooperation. Science (1979) 211, 1390-1396.

10.5840/tpm201467114. 
2. Hamilton, W.D. (1964). The genetical evolution of social behavior. I. J Theor Biol 7, 1-16. 10.4324/9780203790427-4.

3. Ji, T., Xu, J.J., and Mace, R. (2014). Intergenerational and Sibling Conflict Under Patrilocality: A Model of Reproductive Skew Applied to Human Kinship. Human Nature 25, 66-79. 10.1007/s12110-0139188-6.

4. Thomas, M.G., Ji, T., Wu, J., He, Q., Tao, Y., and Mace, R. (2018). Kinship underlies costly cooperation in mosuo villages. R Soc Open Sci 5. 10.1098/rsos.171535.

5. He, Q.-Q., Zheng, X.-D., Mace, R., Tao, Y., and Ji, T. (2021). Hamilton's rule and kin competition in a finite kin population. J Theor Biol 529, 110862. 10.1016/j.jtbi.2021.110862.

6. Wu, J.-J., Ji, T., He, Q.-Q., Du, J., and Mace, R. (2015). Cooperation is related to dispersal patterns in Sino-Tibetan populations. Nat Commun 6, 8693. 10.1038/ncomms9693.

7. Ji, T., Wu, J.-J., He, Q.-Q., Xu, J.-J., Mace, R., and Tao, Y. (2013). Reproductive competition between females in the matrilineal Mosuo of southwestern China. Philosophical Transactions of the Royal 
Society B: Biological Sciences 368, 20130081.

10.1098/rstb.2013.0081.

8. Ji, T., Zheng, X.-D., He, Q.-Q., Wu, J.-J., Mace, R., and Tao, Y. (2016). Kinship as a frequency dependent strategy. R Soc Open Sci 3, 150632. 10.1098/rsos.150632.

9. Croft, D.P., Weiss, M.N., Nielsen, M.L.K., Grimes, C., Cant, M.A., Ellis, S., Franks, D.W., and Johnstone, R.A. (2021). Kinship dynamics: patterns and consequences of changes in local relatedness. Proceedings of the Royal Society B: Biological Sciences 288, 20211129. 10.1098/rspb.2021.1129.

10. West, S.A., Murray, M.G., Machado, C.A., Griffin, A.S., and Herre, E.A. (2001). Testing Hamilton's rule with competition between relatives. Nature 409, 510-513. 10.1038/35054057.

11. West, S.A., Pen, I., and Griffin, A.S. (2002). Cooperation and competition between relatives. Science (1979) 296, 72-75. 10.1126/science.1065507.

12. Úbeda, F., and Gardner, A. (2010). A model for genomic imprinting in the social brain: Juveniles. Evolution (N Y) 64, 2587-2600. 10.1111/j.1558-5646.2010.01015.x. 
13. Johnstone, R.A., and Cant, M.A. (2008). Sex differences in dispersal and the evolution of helping and harming. American Naturalist 172, 318-330. 10.1086/589899.

14. Kuijper, B., and Johnstone, R.A. (2017). How sex-biased dispersal affects sexual conflict over care. American Naturalist 189, 501-514. 10.1086/691330.

15. Micheletti, A.J.C., Ruxton, G.D., and Gardner, A. (2020). The demography of human warfare can drive sex differences in altruism. Evol Hum Sci 2, 1-15. 10.1017/ehs.2020.5.

16. Micheletti, A.J.C., Ruxton, G.D., and Gardner, A. (2018). Why war is a man's game. Proceedings of the Royal Society B: Biological Sciences 285, 0-8. 10.1098/rspb.2018.0975.

17. Micheletti, A.J.C., Ruxton, G.D., and Gardner, A. (2017). Intrafamily and intragenomic conflicts in human warfare. Proceedings of the Royal Society B: Biological Sciences 284. 10.1098/rspb.2016.2699.

18. Faria, G.S., Varela, S.A.M., and Gardner, A. (2015). Sex-biased dispersal, kin selection and the evolution of sexual conflict. J Evol Biol 28, 1901-1910. 10.1111/jeb.12697. 
19. Faria, G.S., Varela, S.A.M., and Gardner, A. (2019). The social evolution of sleep: Sex differences, intragenomic conflicts and clinical pathologies. Proceedings of the Royal Society B: Biological Sciences 286. $10.1098 / \mathrm{rspb} .2018 .2188$.

20. Gurven, M., Winking, J., Kaplan, H., von Rueden, C., and McAllister, L. (2009). A bioeconomic approach to marriage and the sexual division of labor. Human Nature 20, 151-183. 10.1007/s12110-0099062-8.

21. Trivers, R.L. (1972). Parental Investment and Sexual Selection. In Sexual Selection \& the Descent of Man (Aldine de Gruyter), pp. 136179. 10.1002/ajpa.1330400226.

22. Marlowe, F.W. (2007). Hunting and gathering: The human sexual division of foraging labor. Cross-Cultural Research 41, 170-195. 10.1177/1069397106297529.

23. Gurven, M., and Hill, K. (2009). Why Do Men Hunt? Curr Anthropol 50, 51-74. 10.1086/595620.

24. Haas, R., Watson, J., Buonasera, T., Southon, J., Chen, J.C., Noe, S., Smith, K., Llave, C.V., Eerkens, J., and Parker, G. (2020). 
Female hunters of the early Americas. Sci Adv 6, 1-11. 10.1126/sciadv.abd0310.

25. Dyble, M., Thorley, J., Page, A.E., Smith, D., and Migliano, A.B. (2019). Engagement in agricultural work is associated with reduced leisure time among Agta hunter-gatherers. Nat Hum Behav 3, $792-$ 796. 10.1038/s41562-019-0614-6.

26. M. Milcah Paul \& P. Radha Rani (2017). Gender differences in the workload related to household and farm activities - a review. Int $\mathrm{J}$ Agric Sci Res (Chennai) 7, 591-596.

27. Rahman, R.I. (1986). Seasonality of Workload of Women in Rural Areas of Bangladesh: Some Male-Female Comparison. The Bangladesh Development Studies 14, 123-130.

28. Panter-Brick, C. (1996). Seasonal and sex variation in physical activity levels. Am J Phys Anthropol 100, 7-21. 10.1002/(SICI)10968644(199605)100.

29. Agarwal, B. (1997). "Bargaining" and gender relations: Within and beyond the household. Fem Econ 3, 1-51. $10.1080 / 135457097338799$. 
30. Pickett, W., King, N., Lawson, J., Dosman, J.A., Trask, C., Brison, R.J., Hagel, L., Janssen, I., Dosman, J., Brison, R., et al. (2015). Farmers, mechanized work, and links to obesity. Prev Med (Baltim) 70, 59-63. 10.1016/j.ypmed.2014.11.012.

31. Lee, I.M., Shiroma, E.J., Kamada, M., Bassett, D.R., Matthews, C.E., and Buring, J.E. (2019). Association of Step Volume and Intensity with All-Cause Mortality in Older Women. JAMA Intern Med 179, 1105-1112. 10.1001/jamainternmed.2019.0899.

32. Murphy, S.L. (2009). Review of physical activity measurement using accelerometers in older adults: Considerations for research design and conduct. Prev Med (Baltim) 48, 108-114.

10.1016/j.ypmed.2008.12.001.

33. Lau, E.Y., Dowda, M., Mclver, K.L., and Pate, R.R. (2017). Changes in Physical Activity in the School, Afterschool, and Evening Periods During the Transition From Elementary to Middle School. Journal of School Health 87, 531-537.

34. Boddy, L.M., Noonan, R.J., Kim, Y., Rowlands, A. v., Welk, G.J., Knowles, Z.R., and Fairclough, S.J. (2018). Comparability of children's sedentary time estimates derived from wrist worn 
GENEActiv and hip worn ActiGraph accelerometer thresholds. J Sci Med Sport 21, 1045-1049. 10.1016/j.jsams.2018.03.015.

35. Schaefer, C.A., Nigg, C.R., Hill, J.O., Brink, L.A., and Browning, R.C. (2014). Establishing and evaluating wrist cutpoints for the GENEActiv accelerometer in youth. Med Sci Sports Exerc 46, 826-833. 10.1249/MSS.0000000000000150.

36. Rayens, M.K., and Reed, D.B. (2014). Predictors of depressive symptoms in older rural couples: The impact of work, stress and health. Journal of Rural Health 30, 59-68. 10.1111/jrh.12028.

37. Jones-Bitton, A., Best, C., MacTavish, J., Fleming, S., and Hoy, S. (2020). Stress, anxiety, depression, and resilience in Canadian farmers. Soc Psychiatry Psychiatr Epidemiol 55, 229-236. 10.1007/s00127-019-01738-2.

38. Levine, J.A., Weisell, R., Chevassus, S., Martinez, C.D., Burlingame, B., and Coward, W.A. (2001). The work burden of women. Science (1979) 294, 812-812. 10.1126/science.1064627.

39. Sullivan, O. (2019). Gender inequality in work-family balance. Nat Hum Behav 3, 201-203. 10.1038/s41562-019-0536-3. 
40. Raybould, A., and Sear, R. (2021). Children of the (gender) revolution: A theoretical and empirical synthesis of how gendered division of labour influences fertility. Popul Stud (NY) 75, 1-22. 10.1080/00324728.2020.1851748.

41. Ravi, N., Dandekar, N., Mysore, P., and Littman, M.L.M. (2005). Activity recognition from accelerometer data. Proceedings of the National Conference on Artificial Intelligence 3, 1541-1546. 10.1007/978-3-642-02481-8_120.

42. Mannini, A., and Sabatini, A.M. (2010). Machine learning methods for classifying human physical activity from on-body accelerometers. Sensors 10, 1154-1175. 10.3390/s100201154.

43. Valletta, J.J., Torney, C., Kings, M., Thornton, A., and Madden, J. (2017). Applications of machine learning in animal behaviour studies. Anim Behav 124, 203-220. 10.1016/j.anbehav.2016.12.005.

44. Dieu, O., Mikulovic, J., Fardy, P.S., Bui-Xuan, G., Béghin, L., and Vanhelst, J. (2017). Physical activity using wrist-worn accelerometers: comparison of dominant and non-dominant wrist. Clin Physiol Funct Imaging 37, 525-529. 10.1111/cpf.12337. 
45. Bassett, D.R., Toth, L.P., LaMunion, S.R., and Crouter, S.E. (2017).

Step Counting: A Review of Measurement Considerations and Health-Related Applications. Sports Medicine 47, 1303-1315. 10.1007/s40279-016-0663-1.

46. Marshall, S.J., Levy, S.S., Tudor-Locke, C.E., Kolkhorst, F.W., Wooten, K.M., Ji, M., Macera, C.A., and Ainsworth, B.E. (2009). Translating Physical Activity Recommendations into a PedometerBased Step Goal. 3000 Steps in 30 Minutes. Am J Prev Med 36, 410-415. 10.1016/j.amepre.2009.01.021.

47. Wang, L., Liu, T., Wang, Y., Li, Q., Yi, J., and Inoue, Y. (2017). Evaluation on Step Counting Performance of Wristband Activity Monitors in Daily Living Environment. IEEE Access 5, 13020-13027. 10.1109/ACCESS.2017.2721098.

48. Johnstone, R.A., and Cant, M.A. (2010). The evolution of menopause in cetaceans and humans: The role of demography. Proceedings of the Royal Society B: Biological Sciences 277,3765 3771. 10.1098/rspb.2010.0988.

49. Wu, J.-J., He, Q.-Q., Deng, L.-L., Wang, S.-C., Mace, R., Ji, T., and Tao, Y. (2013). Communal breeding promotes a matrilineal social 
system where husband and wife live apart. Proceedings of the Royal Society B: Biological Sciences 280, 20130010.

10.1098/rspb.2013.0010.

50. Mace, R., and Alvergne, A. (2012). Female reproductive competition within families in rural Gambia. Proceedings of the Royal Society B: Biological Sciences 279, 2219-2227. 10.1098/rspb.2011.2424.

51. Murdock, G.P., and Provost, C. (1973). Factors in the division of labor by sex: a cross-cultural analysis. Ethnology 12, 203-225. $10.2307 / 3773347$.

52. Murdock, G.P. (1967). Ethnographic Atlas: A Summary. Ethnology 6, 109-236. 10.2307/3772751.

53. Korotayev, A. (2003). Form of Marriage, Sexual Division of Labor, and Postmarital Residence in Cross-Cultural Perspective: A Reconsideration. J Anthropol Res 59, 69-89. 10.1086/jar.59.1.3631445.

54. Korotayev, A. (2003). Division of labor by gender and postmarital residence in cross-cultural perspective: a reconsideration. CrossCultural Research 37, 335-372. 10.1177/1069397103253685. 
55. Feng, M. (2010). Matrilineal marriage in Tibetan areas in western Sichuan province. Asian Highlands Perspectives 6, 251-280.

56. Schneider, D.M., and Gough, Kathleen. (1961). Matrilineal kinship / edited by David M. Schneider and Kathleen Gough. D. M. (David M. Schneider and K. Gough, eds. (University of California Press).

57. Gallois, S., and Henry, A.G. (2021). The Cost of Gathering Among the Baka Forager-Horticulturalists From Southeastern Cameroon. Front Ecol Evol 9. 10.3389/fevo.2021.768003.

58. Ainsworth, B.E., Haskell, W.L., Whitt, M.C., Irwin, M.L., Swartz, A.M., Strath, S.J., O’Brien, W.L., Bassett, D.R., Schmitz, K.H., Emplaincourt, P.O., et al. (2000). Compendium of Physical Activities: an update of activity codes and MET intensities. Med Sci Sports Exerc 32, S498-S516. 10.1097/00005768-200009001-00009.

59. Panter-Brick, C. (2002). Sexual division of labor: Energetic and evolutionary scenarios. American Journal of Human Biology 14, 627640. 10.1002/ajhb.10074.

60. Koster, J., Lukas, D., Nolin, D., Power, E., Alvergne, A., Mace, R., Ross, C.T., Kramer, K., Greaves, R., Caudell, M., et al. (2019). Kinship ties across the lifespan in human communities. Philosophical 
Transactions of the Royal Society B: Biological Sciences 374. 10.1098/rstb.2018.0069.

61. Koster, J. (2018). Family ties: The multilevel effects of households and kinship on the networks of individuals. R Soc Open Sci 5. $10.1098 /$ rsos. 172159 .

62. Kings M. Phiri (1983). Some Changes in the Matrilineal Family System among the Chewa of Malawi since the Nineteenth Century. $\mathrm{J}$ Afr Hist 24, 257-274.

63. Kishindo, P. (2011). The marital immigrant. Land, and agriculture: a Malawian case study. African Sociological Review 14, 89-97. 10.4314/asr.v14i2.70238.

64. He, Q.Q., Rui, J.W., Zhang, L., Tao, Y., Wu, J.J., Mace, R., and Ji, T. (2022). Communal breeding by women is associated with lower investment from husbands. Evol Hum Sci. 10.1017/ehs.2022.47.

65. He, Q.Q., Wu, J.J., Ji, T., Tao, Y., and Mace, R. (2016). Not leaving home: Grandmothers and male dispersal in a duolocal human society. Behavioral Ecology 27, 1343-1352. 10.1093/beheco/arw053. 
66. Mace, R. (2013). Cooperation and conflict between women in the family. Evol Anthropol 22, 251-258. 10.1002/evan.21374.

67. Ellis, S., Franks, D.W., Nattrass, S., Cant, M.A., Weiss, M.N., Giles, D., Balcomb, K.C., and Croft, D.P. (2017). Mortality risk and social network position in resident killer whales: sex differences and the importance of resource abundance. Proceedings of the Royal Society B: Biological Sciences 284, 20171313. 10.1098/rspb.2017.1313.

68. Ellis, S., Franks, D.W., Weiss, M.N., Cant, M.A., Domenici, P., Balcomb, K.C., Ellifrit, D.K., and Croft, D.P. (2021). Mixture models as a method for comparative sociality: social networks and demographic change in resident killer whales. Behav Ecol Sociobiol 75. 10.1007/s00265-021-03006-3.

69. Wood, B.M., Harris, J.A., Raichlen, D.A., Pontzer, H., Sayre, K., Sancilio, A., Berbesque, C., Crittenden, A.N., Mabulla, A., McElreath, R., et al. (2021). Gendered movement ecology and landscape use in Hadza hunter-gatherers. Nat Hum Behav 5, 436-446.

10.1038/s41562-020-01002-7. 
70. Pontzer, H., Raichlen, D.A., Wood, B.M., Emery Thompson, M., Racette, S.B., Mabulla, A.Z.P., and Marlowe, F.W. (2015). Energy expenditure and activity among Hadza hunter-gatherers. American Journal of Human Biology 27, 628-637. 10.1002/ajhb.22711.

71. Sayre, M.K., Pontzer, H., Alexander, G.E., Wood, M., Pike, I.L., Mabulla, A.Z.P., Raichlen, D.A., Ge, A., Bm, W., II, P., et al. (2020). Ageing and physical function in East African foragers and pastoralists. Philosophical Transactions of the Royal Society B 375, 20190608.

72. Bassett, D.R., Wyatt, H.R., Thompson, H., Peters, J.C., and Hill, J.O. (2010). Pedometer-measured physical activity and health behaviors in U.S. adults. Med Sci Sports Exerc 42, 1819-1825. 10.1249/MSS.0b013e3181dc2e54.

73. Althoff, T., Sosič, R., Hicks, J.L., King, A.C., Delp, S.L., and Leskovec, J. (2017). Large-scale physical activity data reveal worldwide activity inequality. Nature 547, 336-339. 10.1016/j.chieco.2010.05.007. 
74. West, S.A., Griffin, A.S., and Gardner, A. (2007). Evolutionary explanations for cooperation. Current Biology 17, 661-672. 10.1016/j.cub.2007.06.004.

75. Hatchwell, B.J., and Komdeur, J. (2000). Ecological constraints, life history traits and the evolution of cooperative breeding. Anim Behav. 10.1006/anbe.2000.1394.

76. Yuan Chen, Erhao Ge, Liqiong Zhou, Juan Du, R.M. (2022). Femalebiased sex ratios generated by monastic celibacy increase gender inequality in workloads.

77. Trost, S.G., Mciver, K.L., and Pate, R.R. (2005). Conducting accelerometer-based activity assessments in field-based research. Med Sci Sports Exerc 37, S531-S543.

10.1249/01.mss.0000185657.86065.98.

78. Rimbach, R., Yamada, Y., Sagayama, H., Ainslie, P., Anderson, L., Anderson, L., Arab, L., Baddou, I., Bedu-Addo, K., Blaak, E., et al. (2022). Total energy expenditure is repeatable in adults but not associated with short-term changes in body composition. Nat Commun 13, 99. 10.1038/s41467-021-27246-z. 
79. Wahl, Y., Düking, P., Droszez, A., Wahl, P., and Mester, J. (2017). Criterion-validity of commercially available physical activity tracker to estimate step count, covered distance and energy expenditure during sports conditions. Front Physiol 8. 10.3389/fphys.2017.00725.

80. Madimenos, F.C., Snodgrass, J.J., Blackwell, A.D., Liebert, M.A., and Sugiyama, L.S. (2011). Physical activity in an indigenous Ecuadorian forager-horticulturalist population as measured using accelerometry. American Journal of Human Biology 23, 488-497. 10.1002/ajhb.21163.

81. Ward, D.S., Evenson, K.R., Vaughn, A., Rodgers, A.B., and Troiano, R.P. (2005). Accelerometer use in physical activity: best practices and research recommendations. Med Sci Sports Exerc 37, S582588. 10.1249/01.mss.0000185292.71933.91.

82. Sadaf, T., and Siegmann, K.A. (2006). Gendered livelihood assets and workloads in Pakistan's North-West Frontier Province (NWFP). In Troubled times: sustainable development and governance in the age of extremes (SAMA Publications), pp. 1-15. 
83. Paradiso, C., Colino, F., and Liu, S. (2020). The validity and reliability of the mi band wearable device for measuring steps and heart rate. Int J Exerc Sci 13, 689-701.

84. Kuznetsova, A., Brockhoff, P.B., Bojesen, R.H., and Christensen, R.H.B. (2015). ImerTest: Tests in Linear Mixed Effects Models. J Stat Softw 82, 1-26.

85. Wheeler, B., and Torchiano, M. (2010). Package 'ImPerm' August. https://cran.r-project.org/package=ImPerm.

86. Pinheiro, J. (2011). nlme: linear and nonlinear mixed effects models. R package version 3.1-98. http://cran.r-project.org/package=nlme.

87. Bartón, K. (2014). MuMln: Multi-model inference. https://cran.rproject.org/package=MuMIn .

88. Wickham, H., Chang, W., Henry, L., Pedersen, T.L., and Takahashi, K. (2022). Package "ggplot2."

89. Sumner, M., Cook, I., and Baston, D. (2022). Package "sf."

90. Hlavac, M. (2022). Package "stargazer."

91. Bolker, B.M., Brooks, M.E., Clark, C.J., Geange, S.W., Poulsen, J.R., Stevens, M.H.H., and White, J.S.S. (2009). Generalized linear mixed 
models: a practical guide for ecology and evolution. Trends Ecol Evol 24, 127-135. 10.1016/j.tree.2008.10.008.

92. Lo, S., and Andrews, S. (2015). To transform or not to transform: using generalized linear mixed models to analyse reaction time data. Front Psychol 6, 1-16. 10.3389/fpsyg.2015.01171.

93. Ives, A.R. (2015). For testing the significance of regression coefficients go ahead and log-transform count.pdf. Methods Ecol Evol 6, 828-835.

94. Gregoire, T.G., Lin, Q.F., Boudreau, J., and Nelson, R. (2008). Regression Estimation Following the Square-Root Transformation of the Response. the Society of American Foresters 54, 597-606. 